\title{
A GTC STUDY OF THE AFTERGLOW AND HOST GALAXY OF THE SHORT-DURATION GRB 100816A
}

\author{
D. Pérez-Ramírez ${ }^{1}$, J.P. Norris ${ }^{2}$, J. Gorosabel ${ }^{3,4,5}$, A.J. Castro-Tirado ${ }^{3}$, \\ L. Hernández-García ${ }^{3}$, A. de Ugarte Postigo $^{3}$, S. Guziy ${ }^{6}$, J.C. Tello ${ }^{3}$, \\ R. Sánchez-Ramírez ${ }^{3}$ and P. Ferrero ${ }^{7}$
}

\begin{abstract}
We present the results from an optical monitoring campaign aimed at studying the afterglow properties of the short GRB 100816A. We implemented a new way of processing the Swift-BAT data, and based on it we reclassified this burst as short, discarding the initial classification as long. Observations were carried out mainly with the GTC Telescope within the four following days after the burst to investigate the optical photometry of its afterglow, and a year later to localize the host. We completed the optical imaging with the $1.23 \mathrm{~m}$ and $3.5 \mathrm{~m}$ CAHA Telescopes. We built and fitted the nIR-optical SED for the characterization of the host. The best fit of the SED $\left(\chi^{2} /\right.$ d.o.f. $\left.=1.656\right)$ obtained for assumed values of a solar metallicity, and an extinction of $\mathrm{A}_{V}=0.2 \mathrm{mag}$ is obtained for a starburst galaxy with a dominant stellar population aging about 360 Myr.
\end{abstract}

\section{Swift detection of GRB 100816A}

GRB 100816A was detected by Swift-BAT (Oates et al. 2010) on the 16th August 2010. The initial estimated $\mathrm{T}_{90}$ duration $(15-350 \mathrm{keV})$ was $2.9 \pm 0.6 \mathrm{~s}$. A preliminary classification for this GRB based on this parameter and spectral lag analysis although inconclusive pointed out to a long burst (Kouveliotou et al. 1993).

\footnotetext{
1 Universidad de Jaén, Campus Las Lagunillas, s/n, Jaén, Spain

2 Physics Department, Boise State University, Boise, ID 83725, USA

3 IAA-CSIC, Glorieta de la Astronomía s/n, 18008 Granada, Spain

4 Unidad Asociada CSIC-UPV/EHU, Dpto de Física Aplicada I, ETSI, 48013 Bilbao, Spain

5 Ikerbasque, Basque Foundation for Science, Alameda de Urquijo 36-5, 48008 Bilbao, Spain

${ }^{6}$ Nikolaev National University, Nikolaev, Ukraine

7 Instituto de Astrofísica de Canarias, C/ vía Láctea, s/n, Tenerife, Spain
} 
Assuming a redshift of $z=0.8049$ (Tanvir et al. 2010) and a standard cosmology model, the isotropic energy release was estimated to be $\mathrm{E}_{i s o}=(5.8 \pm 0.7) \times$ $10^{51} \mathrm{erg}$, and the peak luminosity $\left(\mathrm{L}_{\text {iso }}\right)_{\max }=(7.3 \pm 1.3) \times 10^{51} \mathrm{erg} \mathrm{s}^{-1}$ (Golenetskii et al. 2010).

\section{The Swift-BAT data analysis reveals a short burst}

We implemented a new approach in the processing of the Swift-BAT data following Norris et al. $(2010,2011)$ procedures. We utilized the raw event data with better statistics (with about 3 times more count rate with non-tagged data than the mask-tagged data) and fitted locally the background at two time intervals. We found a duration over the same energy range $\mathrm{T}_{90}=1.99 \pm 0.02$ ( $1 \sigma$ error). Figure 1 illustrates the Swift-BAT time profile summed over the canonical energy range $15-350 \mathrm{keV}$ and binned to $8 \mathrm{~ms}$ resolution. The use of a finer binning did not reveal any additional significant feature. The burst basically consists of one episode of continuous, mostly smooth emission accompanied with several overlapping substructures with widths as narrow as $\sim 100 \mathrm{~ms}$. We also included in the analysis the spectral lag considerations that together with duration makes GRB 100816A a candidate to short burst. More details on the procedure and Swift-BAT data analysis in Pérez-Ramírez et al. (2013).

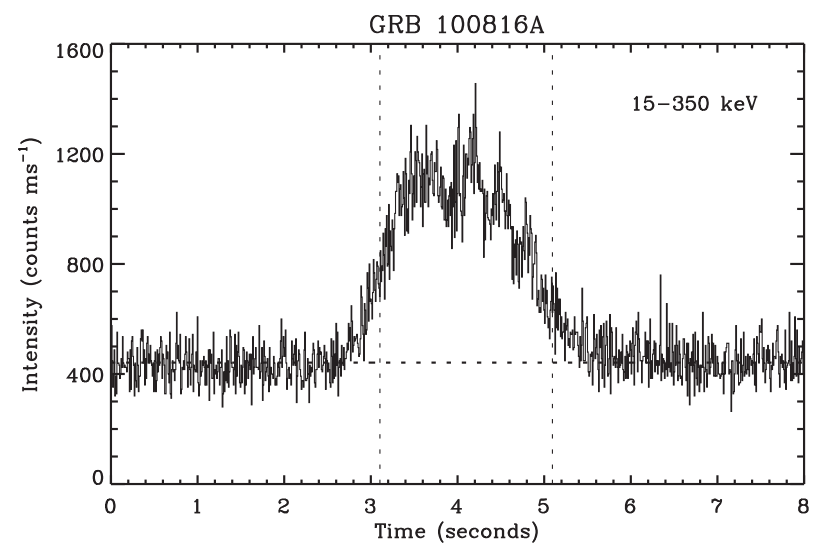

Fig. 1. The Swift-BAT time profile of GRB 100816A summed over the canonical energy range $15-350 \mathrm{keV}$ and binned to $8 \mathrm{~ms}$ resolution.

\section{Optical lightcurve for the GRB 100816A afterglow using the GTC and CAHA Telescopes}

Once the Swift-UVOT detected the presence of an optical afterglow (OA), we started a monitoring campaign in two fronts: in the inmediate timescale, i.e. 

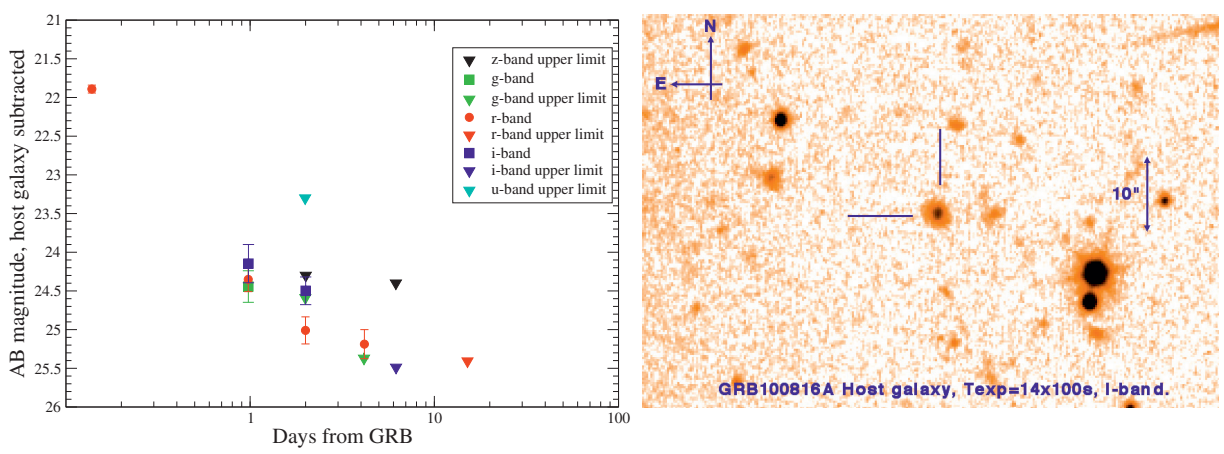

Fig. 2. a) The GTC optical light curve once the host galaxy contribution has been subtracted. b) $i$-band GTC deep image showing the host for GRB 100816A obtained the 8th July 2011.

minutes after the burst when we were able to activate the modest but remotely available $1.23 \mathrm{~m}$ CAHA Telescope, obtaining early observations of the OA (0.14 hours after the burst, Terrón et al. 2010). Later on, we carried out ugriz observations with the $10.4 \mathrm{~m}$ GTC equiped with OSIRIS. We observed the complete data set in the BVRI filters at $1.23 \mathrm{~m}$ CAHA Telescope. For a series of four nights, we observed the OA with the $10.4 \mathrm{~m}$ GTC and were able to obtain the complete lightcurve shown in Figure 2a. The OA appears in the $r$ band about 22 mag hours after the burst, decreasing up to 25.5 within the following four days.

\section{The nIR-optical SED for the GRB 100816A host galaxy}

The host galaxy for GRB 100816A was detected a year later in a deep $i$-band image with the $10.4 \mathrm{~m} \mathrm{GTC}$ (Fig. 2b). We built the Spectral Energy Distribution (SED) for the GRB 100816A based on the photometric ugriz points obtained with the $10.4 \mathrm{~m}$ GTC plus an additional nIR point, in the $H$-band, observed with the $3.5 \mathrm{~m}$ CAHA Telescope. The fit of the SED provides information on the stellar population age, the stellar mass, and on the host galaxy absolute luminosity. We based our SED fitting analysis on templates constructed adopting the metallicity derived from spectroscopic study $\left(\mathrm{Z}=Z_{\odot}\right)$. We mainly utilized synthetic templates, but checks have also been performed using observational templates (Kinney et al. 1996). The synthetic SED analysis is based on the code HyperZ (Bolzonella et al. 2000). The construction of the HyperZ templates was performed using the GALAXEV public code (Bruzual \& Charlot 2003). Figure 3 shows our photometric points and the best fit obtained for assumed values of a solar metallicity, and an extinction of $\mathrm{A}_{V}=0.2 \mathrm{mag}$. The best fit $\left(\chi^{2} /\right.$ d.o.f. $\left.=1.656\right)$ is obtained for a starburst galaxy with a dominant stellar population aging about $\sim 0.36$ Gyr. More details on the host in Pérez-Ramírez et al. (2013). 


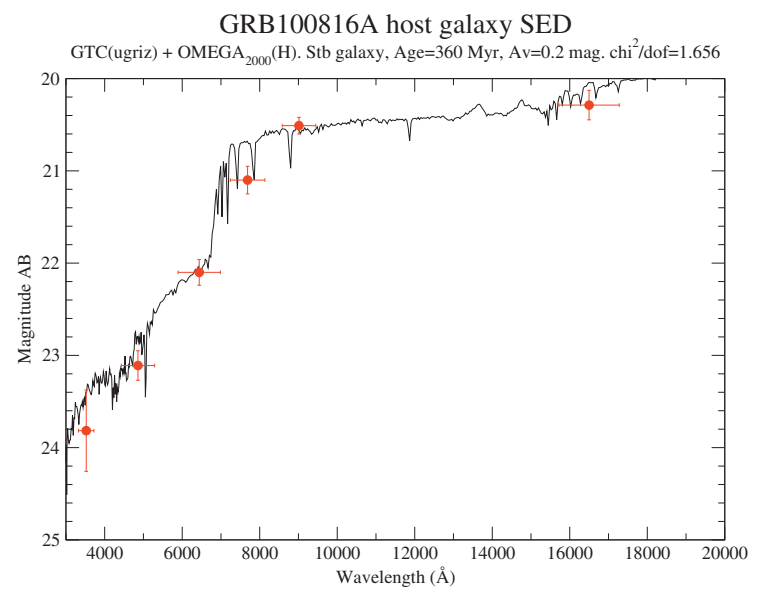

Fig. 3. The nIR-optical SED for the GRB 100816A host galaxy.

\section{Conclusions}

We present the results from an optical monitoring campaign with the $1.23 \mathrm{~m}$ and $3.5 \mathrm{~m}$ CAHA Telescopes, and the $10.4 \mathrm{~m}$ GTC Telescope aimed at studying the afterglow properties of the short-hard GRB 100816A. The GTC campaign was carried out within the four following days after the burst to investigate the optical photometry of the afterglow, and a year later, to localize the host. We built and fitted the nIR-optical SED for the characterization of the host, obtaining the best fit for a starburst galaxy with a dominant stellar population aging about $360 \mathrm{Myr}$, assuming values of a solar metallicity, and an extinction of $\mathrm{A}_{V}=0.2 \mathrm{mag}$. We implemented on this work a new approach for the Swift-BAT data processing and conclude that GRB 100816A is a candidate to short burst. However, the galaxy specification and galaxy type together with inconclusive result on lag, and soft spectrum do not point out to the short nature of GRB 100816A. Comprehensive investigations are being carried out on the morphological nature of the event.

DPR acknowledges support by the Universidad de Jaén and the Spanish program AYA201239727-C03-01 (MINECO). JGU acknowledges support by the Unidad Asociada UPV/EHUIAA/CSIC and the Ikerbasque Foundation for Science.

\section{References}

Bolzonella, M., Miralles, J.-M., \& Pelló, R., 2000, A\&A, 363, 476

Bruzual, G., \& Charlot, S., 2003, MNRAS, 344, 1000

Golenetskii, S., Aptekar,R., Frederiks, D., et al., 2010, GCN Circ 11127

Kinney, A.L., Calzetti, D., Bohlin, R.C., et al., 1996, ApJ, 467, 38 
Kouveliotou, C., Meegan, C.A., Fishman, G.J., et al., 1993, ApJ, 413, L101

Norris, J.P., Gehrels, N., \& Scargle, J.D., 2010, ApJ, 717, 411

Norris, J.P., Gehrels, N., \& Scargle, J.D., 2011, ApJ, 735, 23

Oates, S., Barthelmy, S.D., Beardmore, A.P., et al., 2010, GCN Circ 11102

Pérez-Ramírez, D., Gorosabel, J., Castro-Tirado, A.J., et al., 2013, in preparation

Tanvir, N.R., Vergani, S., Hjorth, J., et al., 2010, GCN Circ 11123

Terrón, V., Fernández, M., Castro-Tirado, A.J., et al., 2010, GCN Circ 11112 
